\title{
Perfil dos cuidadores de idosos nas instituições de longa permanência de Belo Horizonte, M G
}

\author{
Profile of caregivers of elderly \\ in long-term care institutions in Belo Horizonte - M G
}

M arco Túlio deFreitas Ribeiro ${ }^{1}$

Raquel Conceição Ferreira ${ }^{2}$

Efigênia Ferreira eFerreira ${ }^{1}$

Cláudia Silami de M agalhães ${ }^{1}$

Allyson Nogueira M oreira ${ }^{1}$

1 Departamento de

Odontologia Social e

Preventiva, Faculdade de

Odontologia, UFM G. Av.

Antônio Carlos 6627,

Pampulha. 31270-901 Belo

HorizonteM G.

mtuliofr@ig.com.br

${ }^{2}$ Departamento de

Odontologia,

UNIMONTES.
Abstract Thepurpose of thiscross-sectional study was to determine the profile of caregi vers of elderly in long-term care facilities. The studied population included 181 randomly selected caregivers, 98 from philanthropic and 83 from private institutions in the city of Belo Horizonte. Data were collected applying structured questionnaires. The variables evaluated were sex, age group, educational level, marital status, income, economic condition, time of work in the facility, and professional experience. The data were compared using Chi-square and Fisher's exact tests. There was no statistically significant difference between caregivers from the two facilities with regard to sex ( $p=0.62$ ) and income (0.77). In the philanthropic facilities we observed more caregivers aged 50 years or more, widowers, with less than 4 years of study, socio-economic level $D$ and who wereworking as car egivers in the facility for more than two years $(p<0,05)$. The results of this study are relevant for discussing developing and implementing public policies aimed at qualifying the care givers in long-term care institutions for elderly in Belo Horizonte.

Key words Caregivers, Homefor theelderly, Aged
Resumo Estetrabalho avaliou o perfil dos cuidadores de idosos das instituições de longa permanência filantrópicas e privadas de Belo H orizonte - MG. Uma amostra de 181 cuidadores, 98 em filantrópicas e 83 em privadas, foi aleatoriamente sel ecionada e submetida à entrevista estruturada, após assinatura do termo de consentimento livree esclarecido. As variáveis avaliadas foram gênero, faixa etária, escolaridade, estado civil, remuneração mensal (em termos de salários mínimos), condição econômica, tempo de trabalho na instituição etempo na função decuidador. A comparação entre as modalidades de instituições foi realizada pelos testes qui-quadrado e exato de Fisher. Não houve diferença estatística significativa entre as modalidades de instituições quanto ao gênero $(p=0,62)$ e remuneração mensal $(0,77)$ dos cuidadores. Nas instituições filantrópicas, foi observado maior número decuidadores com mais de 50 anos, com estado civil viúvo, com menos de quatro anos de estudo, de classe econômica $D$ e que trabalhavam nas instituições e atuavam como cuidadores há mais de dois anos $(p<0,05)$. Os resultados deste trabalho são relevantes para discussão elaboração e implementação de políticas públicas voltadas à capacitação dos cuidadores de idosos das instituições de longa permanência de Belo H orizonte. Palavras-chave Cuidadores, Asilos para idosos, Idoso 
Introdução

O número de indivíduos idosos vem aumentando na população brasileira ${ }^{1}$, fenômeno acompanhado por uma série de conseqüências sociais. Dentre os desafios a serem vencidos frente às mudanças do perfil demográfico de uma população está a provisão de cuidados de qualidade para idosos com diferentes condições funcionais, econômicas e sociais².

O Brasil, a exemplo de outros países, tem valorizado a manutenção dos idosos em seus domicílios, recebendo cuidados de sua família. Esta estratégia, dentre outros objetivos, visa re duzir custos com assistência hospitalar e instituições asilares. Por outro lado, demanda a disponibilidade deum parente para assistir a necessidade do idoso dependente. Assim, a factibilidade desta proposta deve ser vista de forma crítica, pois é necessário considerar a estrutura familiar, social e cultural do idoso ${ }^{3}$. Entretanto, apesar dessa valorização, percebe-se uma tendência de aumento das taxas de institucionalização, aparecendo como causas desse fenômeno mudanças na estrutura familiar (como redução do número de filhos) e nível de dependência dosidosos².

Ao priorizar a manutenção dosidosos dependentes em seus domicílios, restringe-se o problema principalmenteàs famílias ${ }^{3}$, reduzindo-se, assim, a visibilidade de um crescente problema social. Porém, esta condição transcende a dimensão familiar. É também uma questão pública, quedeve ser foco de políticas sociais, respondendo às necessidades de cuidados, em especial daqueles que dependem do Sistema Único de Saúde (SUS).

Segundo o M inistério da Saúde 5 , 40\% da população idosa necessitam de auxílio para realizar pelo menos uma atividade instrumental de vida diária (AIVD), sendo que desses, 10\% necessitam de ajuda com as atividades básicas de vida diária (AVD). As pesquisas envolvendo idosos institucionalizados mostram que este grupo é formado prioritariamente por mulheres ${ }^{6-8}$, de baixa escolaridade ${ }^{9}$, apresenta altos percentuais de demência ${ }^{10}$, dependência mental ${ }^{10-12}$ e físi$\mathrm{ca}^{7,9,11,12}$. São ainda observadas diferenças quanto à condição funcional em função da modalidade deinstituição: filantrópica ou privada ${ }^{8,12}$. Gorzoni \& Pire ${ }^{10}$ citaram como causas deinstitucionalização dos idosos demenciados o tempo prolongado da doença, o alto grau de dependência, a alta prevalência de distúrbios comportamentais, familiar responsável com mais de 60 anos, a sobrecarga financeira e emocional de esposas e filhas. Segundo estes autores, estes idosos encon- tram-se em estágios mais avançados da síndrome, são mais velhos, mais dependentes e normalmente contam com pouca ou nenhuma assistência familiar. Este quadro chama atenção quanto à humanização na atenção aos idosos fragilizados e/ou institucionalizados ${ }^{4}$.

A necessidade de recursos humanos capacitados para a atenção à saúde do idoso é uma das diretrizes da Política N acional de Saúde do Ido$\mathrm{so}^{13}$ (PNSI). Os cuidadores estão entre os profissionais a serem capacitados e têm um importante papel em auxiliar os idosos nas adaptações físicas e emocionais necessárias para o autocuidado. 0 artigo 30 da portaria interministerial 5.153 determinou a elaboração de protocolos para capacitação de diferentes modalidades de cuidadores: familiar (formal e informal) e institucional. Um ambiente (recursos físicos e pessoais) responsivo e adequado ao desempenho funcional e competência comportamental dos idosos os tornam adaptados, contribuindo assim para o seu bem-estar ${ }^{14}$.

Para Houaiss ${ }^{15}$, cuidador é aquele que se mostra zeloso, diligente para com outrem. Duarte $^{16}$ descreve o cuidador como o profissional que convive diariamente com o idoso, prestando-Ihe cuidados higiênicos, ajudando com a alimentação, administrando medicação e estimulando-o com as atividades reabilitadoras, interagindo, assim, com a equipeterapêutica. Segundo essa autora o cuidador pode ser uma pessoa dafamília ou amigo (cuidador informal) ou uma pessoa contratada para executar essas tarefas (cuidador formal).

0 auxílio prestado ao idoso pode ser classificado segundo o tipo de ajuda (material, instrumental, socioemocional ou cognitivo-informativo), os domínios da ajuda (AIVD e/ou AVD), a intensidade ou quantidade de ajuda, ao local em que ocorre a ajuda (dentro ou fora de casa) e quanto aos padrões temporais envolvidos ${ }^{17}$. Nas necessidades de suporte relativas às AIVD, se enquadram atividades como fazer compras, administrar as contas bancárias e relativas às AVD, tomar banho, vestir-sesozinho, alimentar-sesem ajuda, dentre outras. Em grande parte dos casos, o suporte ao idoso aumenta com o tempo. Além disso, o apoio que o cuidador presta ao idoso é complexo, dificilmente enquadrando-se em somente uma classificação, principalmentenos casos de alto nível de dependência.

Independente do tipo de cuidado prestado, com o tempo essa tarefa torna-se árdua e complexa. Muitas vezes, tal atividade gera sentimento de angústia, insegurança e desânimo. Entretan- 
to, há uma grande variação individual em relação ao sentimento produzido por esse trabalho. Para alguns profissionais, o cuidado aos idosos dependentes podeser inclusiveuma fontedeprazer e conforto, quando se conseguem bons resultados, independente dos esforços físicos ${ }^{18} \mathrm{e}$ psíquicos exigidos ${ }^{19}$. Resultados de um estudo qualitativo ${ }^{20}$ realizado com profissionais da área de enfermagem que cuidavam de idosos dependentes em um serviço de terapia intensiva indicam que, para estes, a ação de cuidar perpassa pelo agir, pela atitude do profissional, sendo de lineada pelas vivências e experiências - valores no decorrer de sua trajetória de vida. Assim, aspectos relativos à inserção social (perfil profissional) vão contextualizar a prática destes profissionais no cuidado a idosos dependentes.

A avaliação da capacitação profissional de pessoas que se dispunham a atuar como cuidadores domiciliares de idosos dependentes, a partir de anúncios de classificados de empregos, em três jornais de grande circulação no país, mostrou que $65,24 \%$ dos candidatos não possuíam qualquer tipo de capacitação para tanto ${ }^{20}$. A falta de qualificação destes profissionais os leva muitas vezes a práticas equivocadas em função de estereótipos associados ao envelhecimento ${ }^{21}$.

Os dados relativos ao perfil dos cuidadores de idosos que trabalham em instituições de longa permanência dentro ${ }^{21-23}$ e fora do Brasil são escassos. Os estudos, na sua maioria, foram realizados com cuidadores informais (familiares e amigos) ${ }^{24-26}$, refletindo a tendência à priorização da manutenção do idoso na comunidade, junto da família. Desta forma, a função de cuidador enquanto prática profissional remunerada permanece obscura, especialmente quanto aos que exercem esta função nas instituições de longa permanência. Entretanto, o estudo dessa população é de suma importância e justificado pelo aumento da população de idosos e o conseqüente crescimento da demanda por instituições de longa permanência. Além disso, informações relativas às pessoas que exercem a função de cuidador enquanto atividade profissional poderão indicar tendências, deficiências enecessidades relativas a este mercado de trabal ho.

Diante do pequeno conhecimento em relação aos cuidadores de idosos formais, este estudo se propôs a avaliar o perfil dos cuidadores de idosos residentes em instituições filantrópicas e privadas de Belo Horizonte - M G quanto ao gênero, nível de instrução, condição econômica, tempo de exercício da função e remuneração segundo o local que exerce a atividade.

\section{M etodologia}

Trata-se de um estudo observacional, do tipo transversal realizado com os cuidadores de idosos das instituições de longa permanência filantrópicas e privadas de Belo Horizonte, M inas Gerais.

Para a realização deste trabalho, foi estabelecida uma parceria entre a Promotoria de Justiça de Defesa da Pessoa Portadora de Deficiência e I dosos (PJDPPDI) e o Colegiado de Pós-Graduação da Faculdade de $O$ dontologia da Universidade de Minas Gerais (FOUFM G), através do credenciamento do pesquisador como voluntário da primeira. 0 vínculo entre essas duas entidades possibilitou ao pesquisador 0 acesso aos dados cadastrais das instituições de Belo H orizonte ea realização do censo dos cuidadores que trabalhavam nas mesmas.

0 universo considerado para este estudo foi de 512 cuidadores. Deste total, 310 cuidadores trabalhavam em instituições definidas pela PJDPPDI como filantrópicas, que possuem entidade mantenedora, e 202 exerciam suas atividades em instituições privadas, que cobram mensalidade de seus residentes.

A amostra foi estimada por meio do método de comparação entreproporções, considerandose um nível de significância de $95 \%(a=0,05)$ e um poder deteste de $90 \%(b=0,10)^{27}$. Os valores considerados para comparação foram os percentuais de cuidadores nas instituições filantrópicas $(60,5 \%)$ e privadas $(39,5 \%)$. A pós a correção para população finita, obteve-se uma amostra de 181 cuidadores (98 em filantrópicas e 83 em privadas). Os cuidadores foram selecionados aleatoriamente através de sorteio sistemático a partir da listagem de cuidadores de cada instituição.

A coleta de dados foi realizada por meio de entrevistas, feitas por um único pesquisador, seguindo rotei ro estruturado previamente testado. As variáveis avaliadas, relativas ao perfil do cuidador, foram gênero, faixa etária, escolaridade, estado civil, remuneração mensal (em termos de salários mínimos), condição econômica, tempo de trabalho na instituição e tempo na função de cuidador.

Para a avaliação econômica dos cuidadores, foi empregado o Critério de Classificação Econômica Brasil ${ }^{28}(A N E P)$, queestima o poder decompra das pessoas e famílias urbanas. Os cuidadores foram categorizados em sete classes econômicas (A1, A2, B1, B2, C, D eE), com base na soma dos escores obtidos considerando o grau de ins- 
trução do chefe da família e a posse e o número dos seguintes itens no seu domicílio: televisor a cores, rádio, banheiro, automóvel, empregada mensalista, aspirador de pó, máquina de lavar, videocassete ou DVD, geladeira efreezer. Por esta classificação, os escores variam de 0 a 34, com os seguintes pontos de corte para cada uma das classes econômicas: A1 (30-34), A2 (25-29), B1 (2124), B2 (17-20), C (11 a 16), D (6-10), E (0-5).

Os dados foram apurados e consolidados em uma planilha do programa M icrosoft Excel. Para a comparação entre os resultados das instituições privadas e filantrópicas, foi utilizado o teste qui-quadrado ou o teste exato de Fisher, dependendo do valor das freqüências observadas e esperadas ${ }^{29}$. Os testes foram realizados empregando o programa Epi Info versão 3.4.

O projeto foi aprovado pelo Comitê de Ética em Pesquisa da Universidade Federal de Minas Gerais (Parecer 212/01). Todos os participantes do estudo e os coordenadores das instituições de longa permanência assinaram o termo deconsentimento concordando em participar da pesquisa.

\section{Resultados ediscussão}

Com relação ao gênero, foi observado que a maior parte dos cuidadores entrevistados era composta por mulheres $(87,8 \%)$, independente da modalidade de instituição: filantrópica ou privada $(p=0,79)$ (Tabela 1$)$. O s resultados obtidos neste estudo foram inferiores aos observados por $\mathrm{M}$ ello ${ }^{23}$, queencontrou 95,3\% demulheres entre os cuidadores de idosos de Porto Alegre edeum estudo qualitativo realizado em São PauIo, onde estas representavam $90 \%$ da amostra. Diversos autores ${ }^{17,25,26}$ observaram maior participação das mulheres nos cuidados familiares de idosos. Muraro \& Boff ${ }^{30}$ descreveram a forte e histórica relação entre fêmeas e prole como origem do cuidado e afetividade da espécie humana. Assim, as raízes históricas e culturais do cuidar podem explicar a expressiva presença feminina observada entre os cuidadores neste estudo. A prática das mulheres em cuidar de seus filhos é um facilitador na adaptação a esta nova atividade. Além disso, a relação afetiva das muIheres com o cuidar pode contribuir na humanização das instituições. Brêtas ${ }^{4}$ relatou que os cuidadores de idosos são majoritariamente mulheres, assumindo esta função por delegação (familiares) ou por necessidade de emprego, vendendo sua força de trabalho cuidando do outro.

Existe uma lacuna na literatura em relação a dados sobreo estado civil dos cuidadores deidosos, impossibilitando a comparação dos resultados obtidos nessa pesquisa. N este trabalho, foi observado que nas instituições privadas mais da metade dos entrevistados (50,6\%) eram casados. Já nas filantrópicas, o percentual de casados foi igual ao de solteiros (37,8\%). Entretanto, o percentual decuidadores viúvos foi significativamente maior nas instituições filantrópicas $(p=0,02)$ (Tabela 1). Esseresultado podeser explicado pela diferente distribuição entre as faixas-etárias dos cuidadores. A maioria dos cuidadores possuía menos de 50 anos em ambas as modalidades. No entanto, houve maior freqüência de cuidadores com mais de 50 anos nas instituições filantrópicas $(20,4 \%)(p=0,00)$. A medida de efeito mostrou que a chance deum cuidador com mais de 50 anos de idade atuar em uma instituição privada é 6,84 vezes menor que em uma filantrópica. Néri \& Sommerhalder ${ }^{17}$ eRicci ${ }^{25}$ et al. observaram maior proporção de indivíduos com mais de50 anos de idadeem uma população de cuidadores familiares. M ello ${ }^{23}$ observou uma média de idade de 36 anos entre cuidadores de instituições privadas de Porto Alegre. Fenkel ${ }^{18}$ et al., em um estudo realizado em instituições do Reino Unido, constataram que poucos cuidadores possuíam mais de 55 anos. Segundo os autores, tal fato deve-se à exigência física dessa função. Assim, a idade éum aspecto importantena atividade de cuidador, pois a dependência dos idosos, principalmente em relação as AVD, demanda esforço físico daqueles que atuam nesta função.

A variável idade pode influir duplamente na atividade de cuidadores de idosos: restringindo 0 acesso dos mais velhos a esse mercado de trabalho elimitando o tempo deatuação destes profissionais na função em decorrência do desgaste físico produzido pela mesma. É importante considerar, entretanto, que profissionais mais experientes podem contribuir em outros aspectos do bem-estar e da qualidade de vida do idoso, uma vez que o cuidado é influenciado por crenças, valores eexperiências vividas na trajetória devida pessoal e profissional ${ }^{20}$. As diferenças entre as modalidades das instituições quanto à faixa etária de seus cuidadores sugerem que para as instituições privadas a idade é um fator na seleção dos profissionais, restringindo a admissão dos mais velhos. Essa afirmativa pode ser reforçada pelo fato de que nas instituições privadas os idosos apresentam maiores índices de dependência.

Os cuidadores das instituições filantrópicas possuem menos anos de estudo $(p=0,00)$. Sabendo-se que pessoas com mais anos de ensino for- 
Tabela 1. Freqüência absoluta e relativa dos cuidadores das instituições privadas e filantrópicas segundo as variáveis gênero, faixa etária, escolaridade, estado civil, remuneração, condição econômica, tempo de trabalho na instituição e tempo na função de cuidador e comparação entre as modalidades por variável avaliada. Belo Horizonte, M inas Gerais, Brasil.

\begin{tabular}{|c|c|c|c|c|c|c|c|c|}
\hline \multirow[t]{2}{*}{ Variáveis avaliadas } & \multicolumn{2}{|c|}{ Total } & \multicolumn{2}{|c|}{$\begin{array}{c}\text { Instituições } \\
\text { filantrópicas } \\
\text { N = 98 }\end{array}$} & \multicolumn{2}{|c|}{$\begin{array}{c}\text { Instituições } \\
\text { privadas } \\
\mathrm{N}=83\end{array}$} & \multirow[t]{2}{*}{$\begin{array}{c}\text { Razão das } \\
\text { chances (IC 95\%) }\end{array}$} & \multirow[t]{2}{*}{$\begin{array}{l}\text { Valor } \\
\text { de p }\end{array}$} \\
\hline & $\mathrm{n}$ & $\%$ & $\mathrm{n}$ & $\%$ & $\mathrm{n}$ & $\%$ & & \\
\hline \multicolumn{9}{|l|}{ Gênero } \\
\hline Feminino & 159 & 87,8 & 85 & 86,7 & 74 & 89,2 & 1 & \\
\hline M asculino & 22 & 12,2 & 13 & 13,3 & 9 & 10,8 & $1,26(0,47-3,41)$ & 0,79 \\
\hline \multicolumn{9}{|l|}{ Estado civil } \\
\hline Solteiro & 70 & 38,7 & 37 & 37,8 & 33 & 39,8 & 1 & \\
\hline Casado & 79 & 43,6 & 37 & 37,8 & 42 & 50,6 & $0,79(0,39-1,57)$ & 0,57 \\
\hline Divorciado & 21 & 11,6 & 14 & 14,2 & 7 & 8,4 & $1,78(0,58-5,61)$ & 0,39 \\
\hline Viúvo & 11 & 6,1 & 10 & 10,2 & 1 & 1,2 & $8,92(1,1-196,2)$ & 0,02 \\
\hline \multicolumn{9}{|l|}{ Faixa etária } \\
\hline$<50$ anos & 158 & 87,3 & 78 & 79,6 & 80 & 96,4 & 1 & \\
\hline$>50$ anos & 23 & 12,7 & 20 & 20,4 & 3 & 3,6 & $6,84(1,82-30,20)$ & 0,00 \\
\hline \multicolumn{9}{|l|}{ Escolaridade - anos de estudo } \\
\hline$M$ ais de 5 & 105 & 58,0 & 39 & 39,8 & 66 & 79,5 & 1 & \\
\hline 0 a 4 & 76 & 42,0 & 59 & 60,2 & 17 & 20,5 & $5,87(2,86-12,46)$ & 0,00 \\
\hline \multicolumn{9}{|l|}{ Condição econômica } \\
\hline B & 40 & 22,1 & 15 & 15,3 & 25 & 30,1 & 1 & \\
\hline C & 91 & 50,3 & 49 & 50,0 & 42 & 50,6 & $1,94(0,85-4,47)$ & 0,12 \\
\hline $\mathrm{D}$ & 49 & 27,1 & 33 & 33,7 & 16 & 19,3 & $3,44(1,31-9,12)$ & 0,00 \\
\hline $\mathrm{E}$ & 1 & 0,5 & 1 & 1,0 & 0 & 0 & Indefinido & 0,39 \\
\hline \multicolumn{9}{|c|}{ Remuneração*(salários mínimos) } \\
\hline$M$ ais de 2 SM & 32 & 17,8 & 16 & 16,5 & 16 & 19,3 & 1 & \\
\hline Até 2 SM & 148 & 82,2 & 81 & 83,5 & 67 & 80,7 & $1,21(0,53-2,77)$ & 0,77 \\
\hline \multicolumn{9}{|c|}{ Tempo de trabalho na instituição } \\
\hline$<5$ anos & 129 & 71,3 & 56 & 57,1 & 73 & 88,0 & 1 & \\
\hline$>5$ anos & 52 & 28,7 & 42 & 42,9 & 10 & 12,0 & $5,47(2,39-12,81)$ & 0,00 \\
\hline \multicolumn{9}{|l|}{ Tempo na função de cuidador } \\
\hline$<5$ anos & 109 & 60,2 & 47 & 47,9 & 62 & 74,7 & 1 & \\
\hline$>5$ anos & 72 & 39,8 & 51 & 52,1 & 21 & 25,3 & $3,20(1,62-6,36)$ & 0,00 \\
\hline
\end{tabular}

*U m cuidador deinstituição filantrópica realizava trabalho voluntário.

valor dep serefere ao teste qui-quadrado com correção deYates ou Exato de Fisher.

mal têm melhor raciocínio lógico, pode-seinferir, a partir dos resultados, que os cuidadores das instituições privadas possuem maior capacidade de auxiliar os idosos em funções mais complexas (AIVD), tais como: auxílio na medicação, receber e transmitir orientações médicas, acompanhar a consultas e ajudar com serviços bancários, recebimento de benefícios e compras. A obtenção de auxílio nestas atividades favorecerá a manutenção da autonomia e bem-estar do idoso. Vale ressaltar que, segundo o M inistério da Saúde ${ }^{5}$, 0 maior percentual de dependência dos idosos se dá em relação as AIVD, conseqüentementea mai- or necessidade de auxílio dos idosos é em relação a estas funções. Portanto, cuidadores com menor formação poderão restringir a autonomia de idosos dependentes para as AIVD.

A diferença entre os cuidadores das duas modalidades de instituição quanto ao nível de escolaridade pode também ser explicada pelo maior número de cuidadores com idade superior a 50 anos (filantrópicas=20,4\%; privadas $=3,6 \%$ ) e de classes econômicas menos favorecidas (classe "D": filantrópicas=33,7\%; privadas $=19,3 \%$ ) nas instituições filantrópicas. Chaimowic $z^{8}$ descreveu que as mulheres mais idosas 
possuem menos anos de ensino formal devido ao menor acesso que as mesmas possuíam a escola, décadas atrás.

$\mathrm{N}$ ão foram encontrados estudos no Brasil que tenham abordado a condição econômica dos cuidadores de idosos, o que dificultou a comparação dos resultados encontrados nessa pesquisa. 0 confrontamento com trabalhos realizados fora do Brasil élimitado por diferenças existentes nos mé todos de avaliação da condição econômica. $\mathrm{Na}$ análise da condição econômica dos cuidadores, no presente trabalho, foram consideradas as categorias B, C, D eE uma vez que, nas duas modalidades de instituição, não houve cuidadores que se enquadrassem na A. O bservou-se diferença entre os cuidadores das duas modalidades de instituições quanto à condição econômica. Nas instituições filantrópicas, houvemaior percentual decuidadores classificados na categoria $\mathrm{D}$ e menor percentual na categoria $B$ comparado às instituições privadas $(p=0,00)$, ainda que não se tenha observado diferenças salariais entre as duas modalidades de instituição. É importante ressaltar que o critério de avaliação econômica utilizado $0^{28}$ considera o domicílio (posse de bens e a escolaridade do responsável) onde a pessoa reside e não a renda individual. Oscuidadores deinstituições privadas encontram-se em uma situação de vantagem em relação aos seus col egas que atuam em instituições filantrópicas. Eles vêm dedomicílios maisbem equipados (maistelevisores, videocasseteseDVD), que podem favorecer o uso de recursos didáticos como fitas de videocassete e DVD na sua formação. Além disto, podem ser mais estimulados na busca de qualificação, pois convivem em ambientes cujos responsáveis são mais instruídos.

A remuneração dos cuidadores de idosos das instituiçõesfilantrópicas e privadas foi semelhante, sendo que "até 2 salários mínimos" foi a faixa salarial mais comum, independentemente da modalidadeda instituição $(p=0,77)$. 0 salário dos profissionais avaliados neste estudo foi inferior ao que Duarte ${ }^{21}$ descreveu para os cuidadores que prestavam assistência a idosos no domicílio. A baixa remuneração pode levar os cuidadores a buscar uma forma de complementação salarial, contribuindo para o estresse destes profissionais.

0 pouco tempo de trabalho dos cuidadores nas instituições pode estar relacionado ao estresse profissional, pois a maioria dos cuidadores trabal hava há menos de cinco anos nestes locais, independentemente da modalidade. Entretanto, nas filantrópicas (42,9\%), o percentual decuidadores que trabalhava há mais de cinco anos foi significativamente maior que nas privadas ( $12 \%)$ $(p=0,00)$. Um dos fatores que pode explicar esta diferença entre os profissionais das duas modalidades de instituições é o fato de que, segundo a literatura, há maior percentual de idosos dependentes nas privadas, exigindo mais dos cuidadores. Um outro aspecto a se considerar é a própria origem das instituições delonga permanência. As instituições para idosos nasceram como serviços para abrigar velhos pobres, sendo geralmentevinculadas a instituições religiosas ${ }^{22}$. 0 surgimento de instituições, enquanto prestadoras de serviços privados, é um fenômeno relativamente recente, decorrente das demandas sociais impostas pelo envelhecimento populacional. Este fato pode explicar também o porquê dos cuidadores de idosos das instituições filantrópicas possuírem maior tempo de experiência profissional $(p=0,00)$.

Os resultados deste estudo mostraram diferenças no perfil dos cuidadores de idosos que podem ter implicações importantes na assistência aos idosos residentes em instituições de longa permanência, especialmentena modalidadefilantrópica. N estas últimas, geralmente vinculadas a instituições religiosas ${ }^{8,11,22}$, os cuidadores são, como nas privadas, principalmente mulheres, porém, há um maior número de viúvas, mais veIhas, com menos anos de ensino formal e menor acesso à informação. Estes dados levam a uma reflexão quanto a diferenças nos critérios de seleção dos profissionais que exercem a função de cuidador nas instituições de longa permanência. Enquanto nas instituições privadas o perfil dos cuidadores segue a lógica de mercado (profissionais mais jovens, mais esclarecidos), nas instituições filantrópicas, geralmente vinculadas a instituições religiosas, outros aspectos podem interferir na seleção e contratação dos cuidadores. É interessante observar que o perfil das cuidadoras das instituições filantrópicas é semelhante ao de seus residentes: mulheres mais velhas, viúvas, pouco instruídas e pobres ${ }^{6,-9}$. Ou seja, perversamente os fatores de exclusão social que promovem a inserção dos idosos nas instituições filantrópicas parecem também determinar a seleção de seus cuidadores. Além dos idosos, as instituições filantrópicas parecem assimilar também pessoas com pouca inserção no mercado de trabaIho formal22. Portanto, diante da importância dos cuidadores para a qualidade de vida dos idosos, é necessária uma atuação intersetorial ${ }^{4}$ ( $M$ inistério da Saúde, da Previdência Social e outros) no sentido de promover efiscalizar a aplicação das políticas públicas direcionadas à capacitação destes profissionais, com maior ênfase para as instituições filantrópicas. 


\section{Conclusões}

De uma maneira geral, o perfil dos cuidadores de idosos em instituições de longa permanência de Belo H orizonteérepresentado maciçamente por profissionais do sexo feminino, com estado civil solteiro ou casado, com menos de 50 anos de idade, de classe econômica $C$, com remuneração mensal de menos de dois salários mínimos, que trabal ham nas instituições e atuam nesta profissão há menos de dois anos.

$N$ as instituições de longa permanência filantrópicas de Belo H orizonte, comparadas às privadas, há maior número decuidadores com mais de 50 anos, com estado civil viúvo, com menos de quatro anos de estudo, com condição econômica menos favorecida eque trabal ham e atuam como cuidadores há mais tempo.

Os resultados deste trabal ho indicam a ne cessidade da discussão, elaboração e implementação de políticas públicas intersetoriais voltadas à capacitação dos cuidadores de idosos das instituições de longa permanência de Belo H orizonte.

\section{Colaboradores}

M TF Ribeiro participou do delineamento do estudo, realizou a coleta de dados, contribuiu na análise e discussão dos resultados e foi o redator principal do artigo; RC Ferreira realizou a análise estatística dos dados e contribuiu na revisão e edição do artigo; CS M agalhães, EF Ferreira eAN M oreira orientaram o delineamento do estudo, acompanharam 0 andamento do trabalho, participaram da análise e discussão dos resultados e contribuíram para a revisão final do artigo.

\section{Agradecimentos}

À Dra. Simone, à assistente social Sônia eà psicóloga Patrícia, da Promotoria de D efesa da Pessoa Portadora de D eficiência Física e do Idoso. 


\section{Referências}

1. Fundação Instituto Brasileiro de Geografia e Estatística. Censo 2000. [acessado 2002 Abr 04]. Disponível em: http://www.ibge.gov.br

2. Vieira EB. Instituições geriátricas: avanço ou retrocesso? Rio de Janeiro: Revinter; 2003.

3. Karsch U. Idosos dependentes: familiares e cuidadores. Cad Saúde Pública 2003; 19(3):861-866.

4. Brêtas ACP. Cuidadores de idosos e o Sistema Ú nico de Saúde. Rev Bras Enferm 2003; 56(3):298-301.

5. Brasil. M inistério da Saúde. [acessado 2005 Nov 25]. Disponível em: http//:www.saúde.gov.br

6. Silva EB. A instituição e o idoso: um estudo das características da instituição e do perfil de seus moradores. Gerontologia 1998; 6:167-176.

7. Telles Filho JCP, Petrilli Filho JF. Causas de inserção de idosos em uma instituição asilar. Escola Ana Nery Revista Enfermagem 2002; 6:135-143.

8. Chaimowicz F, Greco DB. Dinâmica da institucionalização de idosos em Belo Horizonte, Brasil. Rev. Saúde Pública 1999; 33:454-460.

9. M azo GZ, Benedetti TB. Condições de vida dos idosos institucionalizados na Grande Florianópolis. Revista Ciência da Saúde 1999; 18:51-56.

10. Gorzoni ML, Pires SL. Aspectos clínicos da demência senil em instituições asilares. Rev de Psiquiatr Clín 2006; 33(1):18-23.

11. Cunha UGV, Júnior ORB, Siqueira AL. Levantamento epidemiológico psicogeriátrico em asilos. J Bras Psiquiatr 1985; 34:389-394.

12. Waldow VR. Determinação do nível de dependência em enfermagem junto a idosos institucionalizados de Porto Alegre. Rev Gaucha de Enferm 1987; 6:135-143.

13. Brasil. M inistério da Saúde. Política Nacional de Saúde do Idoso. [acessado 2003 Jul 20]. Disponível em: http//www.saúde.gov.br

14. Diogo MJD'E. Formação de recursos humanos. Rev Latino-am Enfermagem 2004; 12(2):280-282.

15. Houaiss A. Dicionário H ouaiss de língua portuguesa. Rio de Janeiro: Objetiva; 2001.

16. Duarte MJRS. Cuidadores? Por que e para quê? Atenção ao idoso no domicílio. Rev Enferm 1996; 34:126-130.

17. Neri AL, Sommerhalder C. As várias faces do cuidado e do bem-estar do cuidador. In: Neri AL, organizadora. Cuidar de idosos no contexto da família: questões psicológicas e sociais. Campinas: Alínea; 2001. p. 9-62.
18. Frenkel HF, Harvey I, Needs K. Oral health care education and its effect on caregiver's knowledge and attitudes: a randomized controlled trial. Community Dent Oral Epidemiol 2002; 30:91-100.

19. Teixeira M H. A saúde do idoso: a arte de cuidar. Rio de Janeiro: EDUERJ; 1998.

20. Brum AK, Tocantins FR, Silva TJES. O enfermeiro como instrumento de ação no cuidar do idoso. Rev Latino-am Enfermagem 2005; 13(6):1019-1026.

21. Duarte YAO. Cuidadores de idosos: uma questão a ser analisada. 0 mundo da saúde 1997; 21:226-230.

22. Martinez SHL. O significado do cuidado para quem cuida do idoso em uma instituição asilar [dissertação]. São Paulo (SP): Escola Paulista de Medicina/ Universidade Federal de São Paulo; 2003.

23. M ello ALSF. Cuidado provido a pessoas idosas residentes em instituições de pequeno porte em Porto Alegre RS: a retórica, a prática e os métodos [dissertação]. Porto Alegre (RS): Faculdade de Odontologia, Universidade Federal do Rio Grande do Sul; 2001.

24. Giacomini KC, Uchoa E, Lima-Costa MFF. Projeto Bambuí: a experiência do cuidado domiciliário por esposas de idosos dependentes. Cad Saúde Pública 2005; 21(5):1509-1518.

25. Ricci NA, Kubota MT, Cordeiro RC. Concordância de observações sobre a capacidade funcional de idosos em assistência domiciliar. Rev. Saúde Pública 2005; 39(5):655-662.

26. Silveira TM, Caldas CP, Carneiro TF. Cuidando de idosos altamente dependentes na comunidade: um estudo sobre cuidadores familiares principais. Cad Saúde Pública 2006; (8):1629-1638.

27. Snedecor GW, Cochran WG. Statistical methods. Ames: Iowa State University; 1989.

28. Associação Nacional de Empresas de Pesquisa. Critério de classificação econômica Brasil.[acessado 2003 M ai 20]. Disponível em: http//www.anep.org.br

29. Soares JF, Siqueira AL. Introdução à estatística médica. Belo Horizonte: COOPMED; 2002.

30. Muraro RM, Boff L. Feminino e masculino: uma nova consciência para o encontro das diferenças. Rio de Janeiro: Sextante; 2002.

Artigo apresentado em 24/04/2006

Aprovado em 17/11/2006 